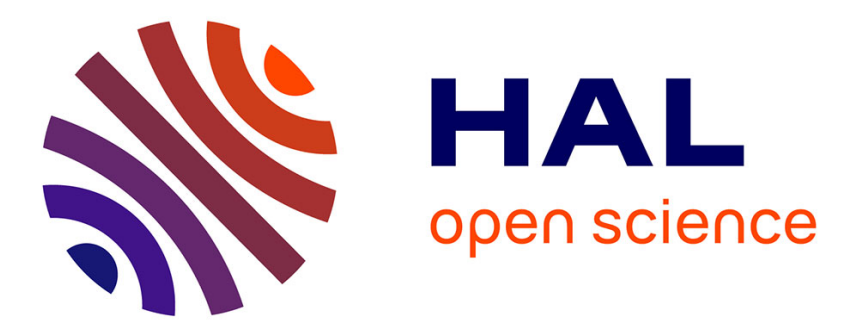

\title{
Development and neuromodulation of spinal locomotor networks in the metamorphosing frog
}

Aude Rauscent, Didier Le Ray, Marie-Jeanne Cabirol-Pol, Keith T Sillar, John Simmers, Denis Combes

\section{- To cite this version:}

Aude Rauscent, Didier Le Ray, Marie-Jeanne Cabirol-Pol, Keith T Sillar, John Simmers, et al.. Development and neuromodulation of spinal locomotor networks in the metamorphosing frog. Journal of Physiology - Paris, 2006, 100 (5-6), pp.317-327. 10.1016/j.jphysparis.2007.05.009 . hal-02347087

\section{HAL Id: hal-02347087 \\ https://hal.science/hal-02347087}

Submitted on 7 Nov 2019

HAL is a multi-disciplinary open access archive for the deposit and dissemination of scientific research documents, whether they are published or not. The documents may come from teaching and research institutions in France or abroad, or from public or private research centers.
L'archive ouverte pluridisciplinaire HAL, est destinée au dépôt et à la diffusion de documents scientifiques de niveau recherche, publiés ou non, émanant des établissements d'enseignement et de recherche français ou étrangers, des laboratoires publics ou privés. 


\title{
Development and neuromodulation of spinal locomotor networks in the metamorphosing frog.
}

Aude RAUSCENT, Didier LE RAY, Marie-Jeanne CABIROL-POL, Keith SILLAR ${ }^{\dagger}$, John SIMMERS and Denis COMBES

Universités Bordeaux 1\&2, CNRS, Laboratoire Mouvement Adaptation Cognition, 33076 Bordeaux, France

${ }^{\dagger}$ School of Biology, University of St Andrews, St Andrews, United Kingdom, KY16 9TS

Corresponding author :

Dr Denis Combes

Universités Bordeaux 2, 1 \& CNRS,

Laboratoire Mouvement Adaptation Cognition, UMR 5227

Bâtiment 2A, 146 rue Léo Saignat, 33076 Bordeaux, France

Phone: (33) 0557574773

Fax: (33) 0556901421

E-mail: denis.combes@ u-bordeaux2.fr

\begin{abstract}
Metamorphosis in the anuran frog, Xenopus laevis, involves profound structural and functional transformations in most of the organism's physiological systems as it encounters a complete alteration in body plan, habitat, mode of respiration and diet. The metamorphic process also involves a transition in locomotory strategy from axial-based undulatory swimming using alternating contractions of left and right trunk muscles, to bilaterally-synchronous kicking of the newly developed hindlimbs in the young adult. At critical stages during this behavioural switch, functional larval and adult locomotor systems co-exist in the same animal, implying a progressive and dynamic reconfiguration of underlying spinal circuitry and neuronal properties as limbs are added and the tail regresses. To elucidate the neurobiological basis of this developmental
\end{abstract}


process, we use electrophysiological, pharmacological and neuroanatomical approaches to study isolated in vitro brain stem/spinal cord preparations at different metamorphic stages. Our data show that the emergence of secondary limb motor circuitry, as it supersedes the primary larval network, spans a developmental period when limb circuitry is present but not functional, functional but co-opted into the axial network, functionally separable from the axial network, and ultimately alone after axial circuitry disappears with tail resorption. Furthermore, recent experiments on spontaneously active in vitro preparations from intermediate metamorphic stage animals have revealed that the biogenic amines serotonin (5-HT) and noradrenaline (NA) exert short-term adaptive control on circuit activity and inter-network coordination: whereas bath-applied 5-HT couples axial and appendicular rhythms into a single unified pattern, NA has an opposite decoupling effect. Moreover, the progressive and region-specific appearance of spinal cord neurons that contain another neuromodulator, nitric oxide (NO), suggests it plays a role in the maturation of limb locomotor circuitry. In summary, during Xenopus metamorphosis the network responsible for limb movements is progressively segregated from an axial precursor, and supra- and intra-spinal modulatory inputs are likely to play crucial roles in both its functional flexibility and maturation.

Keywords: development, locomotion, Xenopus laevis, metamorphosis, spinal motor networks, neuromodulation.

Pages: 25

Figures: 5

Abstract: 311 words 


\section{Introduction}

To survive development and reach maturity, an animal's nervous system must be capable of adapting to continually changing biomechanical and environmental circumstances. Such plasticity in nervous system operation not only requires the assembly of new neuronal circuitry to accommodate additional behavioural demands, but also a dynamic reconfiguration of pre-existing connectivity within maturing networks as well as changes in the integrative electrical properties of constituent neurons. Neuronal networks that generate programmed behaviours such as respiration, locomotion and feeding are useful models for studying developmental correlates of nervous system plasticity since such behaviours can be clearly defined at the neuroethological level, and thus the functional consequences of any developmental alteration in the underlying motor network can be charted with relative accuracy.

The metamorphosis of tadpole to frog in anuran amphibians constitutes one of the most dramatic developmental transformations in biology, involving massive alterations in body structure, a transfer from aquatic to aerial respiration, a switch from herbivorous to carnivorous diet, and biochemical, physiological and morphological changes in virtually all of the animal's organs (Shi, 2000). With respect to locomotion, the alteration in body plan from tadpole to adult is accompanied by the growth of limbs and the regression of the tail as the organism switches its locomotory strategy from the employment of undulatory, tail-based swimming in larvae to limb-based kick propulsion in the young adult. These biomechanical modifications to the locomotory system during metamorphosis require a dynamic anatomical and functional restructuring of underlying neural circuitry within the central nervous system (CNS). Such a remodelling must include the appearance of new sensory and motor pathways to service the emerging limbs, in direct contrast to the concurrent loss of larval spinal circuitry that accompanies 
the regression and eventual elimination of the tail. Therefore the gradual replacement of the tail-based swim network by adult limb-kick circuitry during the metamorphic process implies that addition, deletion and functional reassignment of spinal neuronal elements are occurring simultaneously. However, although Xenopus metamorphosis has been widely studied from endocrinological and molecular biological perspectives (Shi, 2000), the associated developmental changes that occur in actual central neural network function remain largely unknown (but see, e.g. Hoskins, 1990).

Here we review recent evidence from our exploration of in vitro CNS preparations of the clawed toad Xenopus laevis, which continue to produce motor rhythms that share basic features with the patterns that drive real locomotion in vivo. Comparisons of the "fictive" locomotor patterns generated by in vitro preparations at different metamorphic stages have begun to unravel how the developmental remodelling of spinal locomotor circuitry is achieved (Combes et al., 2004), with the eventual aim of determining the neurobiological substrates of such plasticity at the cellular and systems levels. We have also reported that during metamorphosis, neurons that produce the gaseous neuromodulator, nitric oxide (NO), are distributed in a spatial and temporal pattern appropriate for a developmental role in the assembly of the limb motor circuitry (Ramanathan et al., 2006). Preliminary results have also suggested that NO exerts modulatory actions on the expression of spinal locomotor output throughout this period. More recent evidence has shown that the monoamines serotonin and noradrenaline also exert powerful, but opposing, modulatory actions on the expression of spinal locomotor output during metamorphosis, indicating that these signalling molecules may dynamically regulate the short-term operation of spinal locomotor circuitry, in parallel with long-term network development. Finally, comparison is drawn between the 
metamorphic changes in Xenopus and the different strategies utilized by other animals in the ontogeny of their locomotory systems.

\section{Frog metamorphosis}

Anuran metamorphosis involves three major types of developmental process: firstly, a resorption of larval-specific organs such as the gills and tail; second, a de novo formation of adult-specific organs such as the lungs and limbs; and third, a remodelling of pre-existing structures ,such as the liver and intestine, into their adult formats (Dodd and Dodd, 1976; also see Shi, 2000). The entire metamorphic process is orchestrated by thyroid hormones $(\mathrm{TH})$, comprising tetraiodothyronine (thyroxine or $\mathrm{T} 4$ ) which is converted to a more active form, triiodothyronine (T3), by monodeiodinases in target tissues where it regulates gene expression (Denver et al., 1997). Evidence for the causal effects of TH on metamorphosis originally derived from the finding that exogenous T3 and T4 induce precocious metamorphosis (Gudernatsch, 1912), that the inhibition of endogenous T3 synthesis blocks metamorphosis (Dodd and Dodd, 1976), and the close correlation of the normal metamorphic process with elevated plasma concentrations of T3 and T4 (Fig. 1). During pro-metamorphosis (stages 54-58; Nieuwkoop and Faber, 1956), the hindlimbs develop as TH levels rise until a peak is reached some two weeks later at metamorphic climax (stage 59-64). Circulating TH subsequently returns to premetamorphic levels at stage 66 when the tail has completely regressed and the animal employs solely a limb-based mode of locomotion.

A diverse range of changes in nervous system organization and function during metamorphosis has been documented. For example, the bilaterally-paired, giant Mauthner neurons of the medulla involved in larval escape responses undergo degeneration (Kollross, 1981), as do larval-specific mechanoreceptor Rohon-Beard 
neurons (Hughes, 1957). In contrast, postembryonic neurogenesis and proliferation of secondary motoneurons and sensory systems (dorsal root ganglion cells) is required to service the newly developing limbs (Van Mier, 1986). Other types of neuron already present at larval stages, such as cerebellar Purkinje cells, continue to mature and acquire dendritic complexity in accordance with their more complex adult integrative functions (Hauser and Gona, 1984), while still other neuron types, such as trigeminal motoneurons, are reassigned to different jaw muscle targets in the adult frog (Omerza and Alley, 1992).

\section{Developmental segregation of spinal locomotor circuitry}

What general developmental rules allow the metamorphosing frog to first construct and then disengage an adult locomotor network from a functionally distinct and pre-existing tadpole precursor? To address the neural basis of this developmental plasticity, we have established in vitro preparations of the spinal cord and brain stem at different metamorphic stages. Such isolated preparations continue to generate fictive locomotor rhythms spontaneously throughout 2-3 days in vitro (Combes et al., 2004). In intact premetamorphic larvae, as in the earlier postembryonic stages (Roberts et al., 1998), locomotion consists of alternating left-right myotomal contractions that propagate along the body in a rostro-caudal sequence. Although the limb buds appear at stage 49 , undulatory propulsion remains the only mode of locomotion during this developmental period. Consistent with this axial-based swimming behaviour, in vitro recordings from ventral motor roots of the isolated CNS (Fig. 2A1, left) during episodes of fictive locomotion show bilateral alternation of bursts within a given segment, with a brief rostro-caudal delay between segments (Fig. 2A2). 
From the beginning of prometamorphosis at stage 53 until stage 58, the emerging hindlimbs gradually acquire motility, although this consists of alternate abductions/adductions that are often unilateral, apparently to compensate for rolling body movements of the animal. Otherwise, normal locomotion is still exclusively axial, with the hindlimbs that are still insufficiently developed to participate in active propulsion normally being held extended against the body (Fig. 2B1). However, in vitro recordings have revealed that these apparent limb adductions during axial swimming are more complex than a simple tonic activation of the motoneurons innervating hindlimb extensor muscles. Rather, recordings from motor nerve branches to the left and right anterior tibialis (ankle flexor: Flex) and gastrocnemius (ankle extensor: Ext) muscles (see Fig. 2B1 lower) showed that both flexor and extensor motoneurons to a given limb displayed strong rhythmic bursting during fictive axial swimming. It is important to note, however, that this early appendicular rhythmicity, which occurs in strict 1:1 coordination with the axial motor pattern (see boxed cycle in Fig. 2B2), consists of a co-activation of flexor and extensor motoneurons to the same limb, and an alternation between motor bursts in opposite limbs.

From stage 59, at the onset of the metamorphic climax, the limbs are fully functional and they now participate actively in body propulsion (Fig 3A1). Until the tail is resorbed, the animal is still able to employ axial swimming, either alone or in combination with limb-based swimming. In the latter condition, moreover, the two patterns may be expressed independently and with completely different cycle frequencies (Fig. 3A2; see dispersed phase values in circular plot at right) or limb activity may remain co-ordinated 1:1 with the axial rhythm (Fig. 3A3; see tightly concentrated phase values at right). In both cases, and in contrast to the earlier combined pattern during pro-metamorphosis (see Fig. 2B2), the appendicular pattern is 
now coordinated appropriately for generating independent hindlimb kick cycles, with homolateral flexor and extensor motoneurons bursting in alternation, and homologous bilateral motoneurons bursting synchronously. These in vitro results therefore indicate that by metamorphic climax, the hindlimb network is fully functional and generates adultspecific patterns of locomotor output. However, during this critical transitional period, the temporal relationship between the primary axial and secondary limb networks can change spontaneously from decoupled (Fig. 3A2) to coupled (Fig. 3A3) modes of coordination, and vice versa, presumably in response to changing behavioural demands.

Finally, from stage 62, resorption of the tail begins and locomotion becomes exclusively appendicular. As seen in the in vitro recordings of Fig. 3B2 obtained from a now tail-less stage 64 froglet (Fig. 3B1), bouts of rhythmicity expressed in limb motor nerves corresponded to bilaterally-synchronous hindlimb extensions (power-stroke phase of each cycle) that alternate with bipedal flexions (return-stroke phase).

The progressive changes in locomotor strategy that accompany the emergence of the hindlimbs during Xenopus metamorphosis are schematised in Fig. 4. Within a single limb (Fig. 4A), the most striking developmental change in the underlying motor command is the switch from precocious synchronous bursting in homolateral flexor and extensor motoneurons during pro-metamorphosis (Fig. 4A1, see ellipses) to the typical adult pattern at metamorphic climax when these same motoneurons to antagonistic muscles now burst in strict alternation (Fig. 4A2). In terms of the coordination between functionally homologous motoneurons in opposing hindlimbs (Fig. 4B), the opposite transition occurs in keeping with a switch in bilateral limb movement from left-right alternation (Fig. 4B1) to synchronous hindlimb thrusting (Fig. 4B2).

These developmental changes in limb motor activity occur in parallel with a progressive functional separation of limb circuitry from the primary axial network (Fig. 
4C1) until a point (around the metamorphic climax) when the two co-existing circuits can be conjointly active but in rhythms with two completely different frequencies (Fig. 4C2). However, occasionally at this mixed developmental stage, a return to co-ordinated axialand limb-based activity can occur (Fig. 4C3; see experimental recordings in Fig. 3A2, A3), and our recent data suggest that this switch between coupled and decoupled axial and limb motor rhythms may be subject to neuromodulatory control (see below).

Thus at early pro-metamorphic stages, an appendicular motor command appears to exist already, but in an immature form that remains subordinate to the axial network in terms of cycle frequency, homolateral burst synergies, and left/right alternation. This suggests that initially during metamorphosis, the newly-differentiated limb motoneurons and pre-existing axial motoneurons share common synaptic inputs from tail-swimming circuitry. As metamorphosis proceeds, however, the de novo limb-kick circuitry progressively disengages from the faster tail network, becoming increasingly free to operate at its own slower cycle frequency and with a different pattern of burst timing that is appropriate for adult hindlimb control. Thus at later metamorphic stages, the ability of axial- and limb-based motor patterns to be expressed conjointly at very different frequencies (and occasionally independently) is clearly indicative of two co-existing spinal rhythmogenic networks that are approaching a complete functional segregation.

\section{Comparison with rodent locomotor network development}

Striking parallels and differences exist between the developmental changes in limb motor coordination in metamorphosing Xenopus and alterations in appendicular output that occur during the maturation of other vertebrate locomotor systems. In the rat, for example, the acquisition of the adult pattern of locomotion relies on a progressive maturation of spinal circuitry that extends through the pre- and peri-natal 
period. Five days prior to birth (embryonic day 16, E16), the lumbar region of the in vitro rat spinal cord generates a motor pattern that consists of synchronized activity in flexor/extensor motoneurons and on both sides of the cord (Nishimaru and Kudo, 2000; for a review see Vinay et al., 2002). Two days later (at E18.5), however, the hindlimb motor command now displays bilateral alternation, although synchronous bursting still occurs in homolateral flexor and extensor motoneurons. This intermediate developmental coordination therefore strongly resembles the transitional phase in prometamorphic Xenopus (Stage 57-58), where limb motor output during fictive axial swimming also consists of left-right alternation, with flexor and extensor motoneurons on the same side bursting in synchrony (see Fig. 2B2; Fig. 4A1,B1). As the rat approaches birth (at E22), however, the adult-like pattern of motor coordination becomes established in which, unlike in late pro-metamorphic Xenopus where a shift to bilateral synchrony occurs, the newborn rat now expresses both left-right and homolateral flexor-extensor alternation.

Although the mechanisms underlying the developmental changes in Xenopus locomotory network coordination remain to be elucidated, some testable hypotheses can also be formulated from comparison with the early maturation of the rodent locomotory system. In the latter, for example, the transition from in-phase bursting on the two sides of the cord at E16 to left-right alternation in lumbar root bursts at birth (Nishimaru and Kudo, 2000) is thought to derive from a combination of maturational changes, including a decline in widespread electrical coupling and a maturational shift in the equilibrium potential for chloride ions toward more hyperpolarized values. This in turn accounts for a switch in GABA- and glycine-evoked potentials from functional excitation to inhibition (Ben-Ari, 2001) with a resultant transformation of the discharge of 
cross-cord coupled neurons from synchrony to alternation (Nishimaru and Kudo, 2000; Clarac et al., 2003; Kudo et al., 2004).

The left-right alternation of locomotor circuitry in the spinal cord of premetamorphic Xenopus larvae is mediated by inhibitory glycinergic cross-cord connections (Soffe, 1987), as it is in the rat (Cowley and Schmidt, 1995; Kjaerulff and Kiehn, 1997). A reasonable hypothesis therefore is that a weakening (rather than a strengthening, as is likely the case in rodents) of cross-cord glycinergic inhibition occurs in the developing limb circuitry of Xenopus, and in conjunction with an increase (and/or an emergence) of cross-cord excitation, enables network activity to switch from left-right alternation appropriate for axial swimming, to bilateral co-activation necessary for synchronous limb-kicking. This situation is in direct contrast to the requirement for a switch from synchrony to alternation in the activity of homolateral limb flexor and extensor motoneurons. Here the most likely developmental solution is that any electrical coupling between adjacent ipsilateral motoneuron pools becomes weakened in parallel with the de novo formation of intersegmental inhibitory connections to ensure alternating flexor and extensor bursting (see also Kudo et al., 2004).

Finally it has to be remembered that in contrast to the foetal rodent, the limbbased locomotory circuitry of metamorphosing Xenopus is assembled under the dominance of a pre-existing functional system (for tail-based swimming) which initially appropriates the still immature limb network to its own pattern of activity. Thus whereas locomotion in adult mammals derives from the progressive acquisition of functional spinal circuitry from an embryonic (and therefore non-functional) precursor, the emergence of limb-based locomotion in Xenopus is fundamentally different. Here, an already functional locomotor system (for axial swimming) is replaced by a second 
completely different one (for limb-based swimming), with the two systems having to coexist and operate within the same organism while the transition is taking place.

\section{Neuromodulation and locomotor network development}

As for higher vertebrates, descending projections from the brainstem play important roles in both the modulation and maturation of amphibian spinal locomotor networks (for review, see McLean et al., 2000). To date, the best studied supraspinal control pathways in Xenopus are the serotonergic and noradrenergic systems which are present at the time of hatching and originate in two brainstem cell populations, the raphe nucleus (Sillar et al., 1995) and the isthmic region (the amphibian equivalent of the locus coeruleus (Marin et al., 1996)), respectively. The biogenic amines serotonin (5HT) and noradrenaline (NA) have very different short-term modulatory actions on the spinal locomotory circuitry of the hatchling tadpole. Whereas exogenously-applied 5HT increases the duration and intensity of axial motoneuron bursts with little effect on swimming frequency (Sillar and Roberts, 1992), NA slows fictive swimming and reduces burst durations relative to cycle periods (McDearmid et al., 1997). An important common target of this aminergic modulation is the strength of inhibitory synaptic connections within locomotory spinal circuitry, and in particular, the glycinergic inhibition of motoneurons by cross-cord commissural interneurons responsible for reciprocal midcycle inhibition and the left-right alternation necessary for undulatory swimming. Again $5 \mathrm{HT}$ and NA exert opposing modulatory effects, with $5 \mathrm{HT}$ decreasing the amplitude of mid-cycle glycernergic IPSPs during fictive swimming, whereas NA enhances them (McDearmid et al., 1997).

$5 \mathrm{HT}$ has also been implicated in the maturation of the larval locomotory system, and in particular, the developmental acquisition of more efficient and flexible axial 
swimming behaviour. This conclusion derived from previous findings that the early maturation of postembryonic swimming rhythmicity between late embryonic and early larval stages (Sillar and Roberts, 1991) is strikingly similar to the effects of exogenous 5HT described above (Sillar et al., 1992), and that these effects occurred in an agedependent, rostro-caudal fashion in temporal correlation with the progressive ingrowth of serotonergic axons to the spinal cord (Sillar et al., 1993; Sillar et al., 1995; Van Mier, 1986).

Our most recent findings also indicate that monoamines continue to exert important and different regulatory influences on spinal locomotor circuitry during the later metamorphic development of Xenopus. A particularly interesting example is the ability of 5-HT and NA to modulate, again in an opposing manner, the functional coupling between axial- and limb-based locomotor rhythms in animals at metamorphic climax (see Fig. 4C2, 3; Rauscent et al., unpublished observations). In stage 61 in vitro preparations that are spontaneously expressing independent axial and limb rhythms (Fig. 4C2), bath-applied 5-HT (5-10 $\mathrm{MM})$ causes an acceleration of appendicular rhythmicity, a slowing down of axial activity, and a 1:1 coupling of the two otherwise separate patterns into a single, combined rhythm (Fig. 4C3). In contrast, the presence of NA $(10 \mu \mathrm{M})$ has the opposite effect, in that during already spontaneously-coupled locomotor rhythmicity (as in Fig. 4C3), this amine's action is to dissociate the two rhythms by causing a differential increase and decrease, respectively, in the frequency of axial and limb activity. It seems likely that these opposing network-specific effects on cycle rate are accompanied by an amine-dependent increase (for 5-HT) and decrease (for NA) in the strength of synaptic interactions between the two circuits. Presumably such short-term plasticity in the functional relationship between co-existing locomotor networks is designed to satisfy the immediate propulsive requirements of the animal, 
and it now remains to be seen whether 5-HT and/or NA are additionally engaged in the longer-term developmental changes in Xenopus locomotor circuitry during metamorphosis.

Another source of supraspinal influence on Xenopus tadpole locomotor circuitry is the gaseous signalling molecule nitric oxide (NO) which has been implicated in numerous developmental and physiological processes throughout the animal kingdom. NO has also been found to be involved in developmental mechanisms as diverse as neurogenesis, cell proliferation (Kuzin et al., 1996), differentiation (Contestabile and Ciani, 2004) and apoptosis (Pinsky et al., 1999; Zhang et al., 2004). Our recent findings are consistent with an equally multi-faceted role of NO in the metamorphic development of Xenopus locomotory spinal circuitry (Ramanathan et al., 2006). This has been suggested by the progressive pattern of appearance of NO-synthase (NOS) containing neurons within the spinal cord during metamorphosis. Whereas in pre-metamorphic tadpoles, NOS expression is restricted to the brainstem (McLean and Sillar, 2000, 2002), at early stages of pro-metamorphosis (coincident with the emergence of the foreand hindlimb buds), two distinct clusters of spinal NOS-positive neurons interspersed with areas devoid of stained somata are observed. Interestingly, motoneurons innervating the fore- and hindlimb buds (revealed after retrograde labelling with horseradish peroxidase (HRP)) are located exclusively in areas where NOS staining is absent, indicating that during early stages of metamorphosis, nitrergic neurons are excluded from the very cord regions where the new limb circuits are forming. Therefore it is possible that, as reported elsewhere in the Xenopus CNS (Peunova et al., 2001), the rat brain (Moreno-Lopez et al., 2004) and Drosophila (Kuzin et al., 1996), NO may have an early negative influence on network development, so that its absence from the 
cervical and lumbar spinal regions in early pro-metamorphic stages could allow the neuronal proliferation necessary for the initial assembly of limb locomotor circuitry.

However, as Xenopus reaches late pro-metamorphosis, NOS expression is found to be evenly distributed along the entire length of the spinal cord (Ramanathan et al., 2006). Thus NO could now have a completely different regulatory role such as occurs in the developing brains of the chick and rodent, where NO is positively engaged in the late-phase refinement of dendritic topographies (Inglis et al., 1998) and the final adult tuning of synaptic connectivity (Wu et al., 2001).

In addition to a likely developmental role, NO has been found to act as a potent modulator of locomotory rhythmicity in post-embryonic Xenopus tadpoles where it has been shown to decrease the duration and frequency of swimming episodes through both direct and indirect influences at spinal and brainstem levels, respectively (McLean and Sillar, 2000, 2002). Our preliminary data suggest that NO continues to modulate locomotor activity through metamorphosis, albeit in a strikingly different fashion to the hatchling embryo. In contrast to the pre-metamorphic animal where NO has a net inhibitory effect on swimming, exogenously-applied NO to pro-metamorphic spinal cord/brainstem preparations activates both axial and limb motor rhythms, while removal of endogenous NO has the opposite effect (Combes et al., unpublished observations). Presumably this switch in NO's modulatory action reflects developmental alterations in brainstem nitrergic systems and/or in the responsiveness to NO of the downstream spinal networks themselves.

\section{Comparison with other metamorphosing locomotor systems}

It is also instructive to compare the metamorphic changes in locomotory behaviour in anuran amphibians like Xenopus with the developmental transformations that occur in 
other amphibian species as well as in certain insects (Figure 5). It is important to remember that the transition from the anuran tadpole to froglet involves a switch between two completely different locomotor strategies (primary axial-based swimming and secondary limb-based propulsion) while the organism continues to behave in its normal environment (Fig. 5A). Thus at critical stages of metamorphosis, the two propulsive mechanisms together with their underlying neural machinery must operate within the same animal, prior to the adult system progressively superseding the larval system as the latter disappears. This replacement of one already functional system by another in Xenopus contrasts with metamorphosis in urodele amphibians, such as the salamander Pleurodeles waltlii, which also experiences the emergence of limbs in primary axial-swimming larvae and gains the capacity for secondary quadrupedal locomotion. However Pleurodeles and Xenopus differ in two major ways: firstly, the salamander does not lose its tail during metamorphosis and thereby conserves the ability for both axial- and limb-based locomotion in adulthood. Moreover, while the neural machinery for both locomotor strategies co-exist within the adult salamander's spinal cord, in contrast to the metamorphosing Xenopus, the two behavioural modes are rarely co-expressed, with undulatory swimming being used solely in an aquatic environment while quadrupedal walking is employed during terrestrial locomotion (Delvolve et al., 1997; see Figure 5B). Furthermore, computer simulations have suggested that both behaviours may actually derive from different functional configurations of the same basic spinal circuitry (Bem et al., 2003), a likelihood that is supported by a second fundamental difference between Xenopus and Pleurodeles: during walking in the latter, the flexion/extension movements of limbs at the same (scapular or pelvic) girdle operate in left/right alternation (rather than in synchrony as in 
Xenopus adults), in a same manner as the axial musculature during undulatory swimming.

Fundamental differences also exist between the developmental transformations of Xenopus and insect metamorphosis. For example, holometabolous insects such as the moth Manduca sexta, and the fruitfly Drosophila melanogaster, undergo a complete transformation from a crawling larva to a walking/ flying adult (Figure 5C). In contrast to amphibians, this metamorphic transition is mediated by a resting pupal stage in which all locomotory activity ceases. A further important difference is that in Manduca and Drosophila, although the assembly of adult-specific neural circuitry relies to a certain extent on neurogenesis during larval or pupal stages, most adult motoneurons and remaining interneurons derive from a functional and anatomical re-specification of preexisting larval circuitry (Consoulas et al., 2000). This again contrasts with anuran metamorphosis during which primary axial motoneurons and associated spinal circuitry disappear with tail resorption, while entirely new populations of secondary motoneurons and sensory neurons are born to service the emerging limbs (Van Mier, 1986).

Finally, in hemimetabolous insects, such as the locust and cricket (Figure 5D) in which metamorphosis is incomplete and there is no resting pupal phase, most adultspecific motor networks are assembled during embryogenesis, but await appropriate levels of maturation before becoming functional. In the flightless nymphal locust, for example, the central flight motor circuitry is already present and potentially operational, but it is either actively inhibited until the wings become fully developed or awaits permissive modulatory signals that appear in adulthood (Stevenson and Kutsch, 1988). Although evidently different from the metamorphic assembly of limb motor circuitry in Xenopus, an equivalent precocious development has been reported in the bullfrog Rana catesbeiana where functional neural networks responsible for adult aerial respiration are 
already present at gill-breathing premetamorphic stages, but only become active after metamorphosis when a developmental removal of GABAergic inhibition occurs (Straus et al., 2000).

\section{Conclusion}

The establishment of new in vitro preparations of the Xenopus CNS which are capable of generating motor rhythms in the limb and/or tail ventral roots appropriate to drive locomotor movements of the host organism's developmental stage (Combes et al., 2004), has thus permitted initial insights into how the complex metamorphic transition in locomotor strategy is accomplished. In addition, these preparations offer numerous avenues for further studies to address key facets of the neural plasticity accompanying metamorphosis that hitherto have not been experimentally tractable. Since the rise in plasma levels of thyroid hormones is necessary and sufficient to orchestrate the entire metamorphic process, THs are well positioned at the pinnacle of a signalling cascade which, in the spinal cord, triggers neuronal differentiation, synaptogenesis, apoptosis and many other events that allow the new limb circuitry to develop within the framework of an existing axial system. Whilst the signals in this hierarchy are not yet known, one possibility is that THs trigger the expression of NOS in the spinal cord in a regionallyand temporally-specific pattern and that NO then engages an ensemble of subordinate developmental and modulatory pathways that in turn regulate the emergence of the limb circuit and the disappearance of the axial system (Ramanathan et al, 2006). At earlier embryonic stages of tadpole development, around the time of hatching, NO functions as a metamodulator to shift the balance of influence of two aminergic systems with opposing actions on swimming (serotonin and noradrenaline) towards the noradrenergic form of output (McLean and Sillar, 2004). It is therefore of considerable interest that the 
two amines also exert opposing modulatory actions on the expression of tail and limb rhythms during metamorphosis, albeit different influences to those occurring earlier in pre-metamorphic development. Might NO also differentially regulate the interactions between these two spinal locomotory systems via the metamodulation of descending aminergic (and other) pathways?

Another avenue for exploration resides at the molecular developmental level. Much is now known about gene expression and the transcription factors that regulate the differentiation of spinal circuitry and the ontogeny of limb movements in mammals. Similar molecular mechanisms also appear to be engaged during the development of the zebrafish swimming system, suggesting a common embryonic template for spinal motor system assembly. However, the construction of the limb circuitry in Xenopus takes place at comparatively much later stages in development and, rather than the limb and axial spinal networks emerging in parallel, the limb network forms once the axial system is already mature. Is the same palette of molecular signals expressed at these later stages, and how does this secondary developmental programme for the construction of new neuronal types and connections coordinate and interact with the molecular substrate of already existing tail circuitry? Resolving such issues should help provide insights into the developmental assembly and operation of related neural networks for behaviour in general. 


\section{References}

Bem, T., Cabelguen, J.M., Ekeberg, O. and Grillner, S. (2003). From swimming to walking: a single basic network for two different behaviors. Biol Cybern 88, 79-90.

Ben-Ari, Y. (2001). Developing networks play a similar melody. TINS 24, 353-360.

Clarac, F., Brocard, F. and Vinay, L. (2003). The maturation of locomotor networks. Prog. Brain. Res. 143, 57-66.

Combes, D., Merrywest, S.D., Simmers, J. and Sillar, K.T. (2004). Developmental segregation of spinal networks driving axial- and hindlimb-based locomotion in metamorphosing Xenopus laevis. J. Physiol. Lond. 559, 17-24.

Consoulas, C., Duch, C., Bayline, R.J. and Levine, R.B. (2000). Behavioral transformations during metamorphosis: remodeling of neural and motor systems. Brain. Res. Bull. 53, 571583.

Contestabile, A. and Ciani, E. (2004). Role of nitric oxide in the regulation of neuronal proliferation, survival and differentiation. Neurochem Int 21, 903-914.

Cowley, K.C. and Schmidt, B.J. (1995). Effects of inhibitory amino acid antagonists on reciprocal inhibitory interactions during rhythmic motor activity in the in vitro neonatal rat spinal cord. J. Neurophysiol. 74, 1109-1117.

Delvolve, I., Bem, T. and Cabelguen, J.M. (1997). Epaxial and limb muscle activity during swimming and terrestrial stepping in the adult newt, Pleurodeles waltl. J. Neurophysiol. 78, 638-650.

Denver, R.J., Pavgi, S. and Shi, Y.B. (1997). Thyroid hormone-dependent gene expression program for Xenopus neural development. J Biol Chem 272, 8179-8188.

Dodd, M.H.I. and Dodd, G.S. (1976). The Biology of Metamorphosis. In Physiology of the Amphibia, LofTs, ed. (New York: Academic Press), pp. 467-599.

Gudernatsch, J.F. (1912). Feeding experiments on tadpoles. I. The influence of specific organs given as food on growth and differentiation: a contribution to the knowledge of organs with internal secretion. Arch. Entwicklungsmech. Org. 35, 457-483.

Hauser, K.F. and Gona, A.G. (1984). Purkinje cell maturation in the frog cerebellum during thyroxine-induced metamorphosis. Neuroscience 11, 139-155.

Hoskins, S.G. (1990). Metamorphosis of the amphibian eye. J. Neurobiol. 21, 970-989.

Hughes, A. (1957). The development of the primary sensory system in Xenopus laevis. J. Anat. 91, 323-338.

Inglis, F.M., Furia, F., Zuckerman, K.E., Strittmatter, S.M. and Kalb, R.G. (1998). The role of nitric oxide and NMDA receptors in the development of motor neuron dendrites. J. Neurosci. 18, 10493-10501.

Kjaerulff, O. and Kiehn, O. (1997). Crossed rhythmic synaptic input to motoneurons during selective activation of the contralateral spinal locomotor network. J. Neurosci. 17, 94339447.

Kollross, J.J. (1981). In Metamorphosis: A Problem in developmental biology, I, G.L.andE, F., ed. (New York: Plenum Press), pp. 445-459.

Kudo, N., Nishimaru, H. and Nakayama, K. (2004). Developmental changes in rhythmic spinal neuronal activity in the rat fetus. Prog. Brain. Res. 143, 49-55.

Kuzin, B., Roberts, I., Peunova, N. and Enikolopov, G. (1996). Nitric oxide regulates cell proliferation during Drosophila development. Cell 87, 639-649.

Leloup, J. and Buscaglia, M. (1977). La triiodothyronine: hormone de la métamorphose des amphibiens. C. R. Acad. Sci. 284, 2261-2263.

Marin, O., Smeets, W.J. and Gonzalez, A. (1996). Do amphibians have a true locus coeruleus? Neuroreport 7, 1447-1451. 
Mcdearmid, J.R., Scrymgeour-Wedderburn, J.F. and Sillar, K.T. (1997). Aminergic modulation of glycine release in a spinal network controlling swimming in Xenopus laevis. J Physiol 503 ( Pt 1), 111-117.

Mclean, D.L., Merrywest, S.D. and Sillar, K.T. (2000). The development of neuromodulatory systems and the maturation of motor patterns in amphibian tadpoles. Brain. Res. Bull. 53, 595-603.

Mclean, D.L. and Sillar, K.T. (2000). The distribution of NADPH-diaphorase-labelled interneurons and the role of nitric oxide in the swimming system of Xenopus laevis larvae. J Exp Biol 203, 705-713.

Mclean, D.L. and Sillar, K.T. (2002). Nitric oxide selectively tunes inhibitory synapses to modulate vertebrate locomotion. J. Neurosci. 22, 4175-4184.

Moreno-Lopez, B., Romero-Grimaldi, C., Noval, J.A., Murillo-Carretero, M., Matarredona, E.R. and Estrada, C. (2004). Nitric oxide is a physiological inhibitor of neurogenesis in the adult mouse subventricular zone and olfactory bulb. J. Neurosci. 24, 85-95.

Nieuwkoop, P. and Faber, B. (1956). Normal Tables for Xenopus laevis (Amsterdam: North Holland Publishing Company)

Nishimaru, H. and Kudo, N. (2000). Formation of the central pattern generator for locomotion in the rat and mouse. Brain. Res. Bull. 53, 661-669.

Omerza, F.F. and Alley, K.E. (1992). Redeployment of trigeminal motor axons during metamorphosis. J. Comp. Neurol. 325, 124-134.

Peunova, N., Scheinker, V., Cline, H. and Enikolopov, G. (2001). Nitric oxide is an essential negative regulator of cell proliferation in Xenopus brain. J. Neurosci., 8809-8818.

Pinsky, D.J., Aji, W., Szabolcs, M., Athan, E.S., Liu, Y., Yang, Y.M., Kline, R.P., Olson, K.E. and Cannon, P.J. (1999). Nitric oxide triggers programmed cell death (apoptosis) of adult rat ventricular myocytes in culture. Am J Physiol 277, H1189-H1199.

Ramanathan, S., Combes, D., Molinari, M., Simmers, J. and Sillar, K.T. (2006). Developmental and regional expression of NADPH-diaphorase/nitric oxide synthase in spinal cord neurons correlates with the emergence of limb motor networks in metamorphosing Xenopus laevis. Eur. J. Neurosci. 24, 1907-1922.

Roberts, A., Soffe, S.R., Wolf, E.S., Yoshida, M. and Zhao, F.Y. (1998). Central circuits controlling locomotion in young frog tadpoles. Ann N Y Acad Sci 860, 19-34.

Shi, Y.B. (2000). Amphibian metamorphosis; from morphology to molecular biology (NewYork: Wiley-Liss Inc.)

Sillar, K.T. and Roberts, A. (1991). Segregation of NMDA AND NON-NMDA receptors at separate synaptic contacts: evidence from spontaneous EPSPs in Xenopus embryo spinal neurons. Brain Res. 545, 24-32.

Sillar, K.T. and Roberts, A. (1992). Phase-dependent modulation of a cutaneous sensory pathway by glicinergic inhibition from the locomotor rhythm generator in Xenopus embryos. Eur. J. Neurosci. 4, 1022-1034.

Sillar, K.T., Wedderburn, J.F.S. and Simmers, A.J. (1992). Modulation of swimming rhythmicity by 5-hydroxytriptamine during post-embryonic development in Xenopus laevis. Proc. Roy. Soc. Lond. 250, 107-114.

Sillar, K.T., Wedderburn, J.F.S., Woolston, A.M. and Simmers, A.J. (1993). Control of locomotor movements during vertebrate development. News in Physiological Sciences 8, 107-111.

Sillar, K.T., Woolston, A.M. and Wedderburn, J.F. (1995). Involvement of brainstem serotonergic interneurons in the development of a vertebrate spinal locomotor circuit. Proc R Soc Lond B Biol Sci 259, 65-70.

Soffe, S.R. (1987). Ionic and pharmacological properties of reciprocal inhibition in Xenopus embryo motoneurones. J Physiol 382, 463-473. 
Stevenson, P.A. and Kutsch, W. (1988). Demonstration of functional connectivity of the flight motor system in all stages of the locust. J. Comp. Physiol. A 162, 247-259.

Straus, C., Wilson, R.J. and Remmers, J.E. (2000). Developmental disinhibition: turning off inhibition turns on breathing in vertebrates. J. Neurobiol. 45, 75-83.

Van Mier, P. (1986). The development of the motor system in the clawed toad Xenopus laevis. University of Nijmengen, Nijmengen.

Vinay, L., Brocard, F., Clarac, F., Norreel, J.-C., Pearlstein, E. and Pflieger, J.-F. (2002). Development of posture and locomotion: an interplay of endogenously generated activities and neurotrophic actions by descending pathways. Brain. Res. Rev. 40, 118-129.

Wu, H.H., Selski, D.J., El-Fakahany, E.E. and Mcloon, S.C. (2001). The role of nitric oxide in development of topographic precision in the retinotectal projection of chick. J. Neurosci. 21, 4318-4325.

Zhang, Y., Zhang, J. and Zhao, B. (2004). Nitric oxide synthase inhibition prevents neuronal death in the developing visual cortex. Eur. J. Neurosci. 20, 2251-2259.

\section{Acknowledgments}

This work is supported by a doctoral studentship from the Conseil Régional d'Aquitaine

to A. Rauscent, the CNRS ("ATIPE jeune chercheur", France), and a research

interchange grant from the Leverhulme Trust (UK). 


\section{Figure Legends}

Figure 1. Timetable of development in Xenopus laevis and the correlation of changes in thyroid hormone (T4 and T3) concentration with metamorphosis. Plasma $\mathrm{TH}$ is undetectable throughout premetamorphosis, from embryo hatching at stage 35/36 (Nieuwkoop and Faber, 1956) until the onset of metamorphosis at stage 54. TH levels increase during prometamorphosis (stages 54-58) to peak at the climax (stages 59-64) when the major changes in body format occur. THs then decline until the completion of metamorphosis at stage 66 . The entire metamorphic process lasts $\sim 4$ weeks. AU: arbitrary units. (Adapted from Leloup and Buscaglia, 1977).

Figure 2. Fictive swimming in isolated spinal cord/brain stem preparations from pre(A1) and pro-metamorphic (B1) Xenopus tadpoles. A2. Ventral root (VR) recordings from a stage 50 preparation (as in A1) showing typical larval left-right alternating bursts of motor activity (see L- and R-VR6) which occur along the cord with a brief rostrocaudal delay (compare R-VR2, 6 and 17 with dotted line). B2. Ventral root recordings from a stage 58 preparation (as in B1). Motor output to the developing hindlimbs was recorded from left and right flexor (tibialis) and extensor (gastrocnemius) muscle nerve branches, along with an axial (R-VR15) motor root. Axial and limb motor bursts are coordinated in a single rhythm, with homolateral flexor and extensor motoneurons bursting in synchrony, but in alternation with bursts in homologous motoneurons on the opposite side of the cord.

Figure 3. Fictive swimming in isolated spinal cord/brain stem preparations from a tadpole at metamorphic climax (A1) and a young adult (B1). A2, A3. Appendicular 
network activity in a stage 61 animal (as in A1) showing typical bilateral co-activation of flexor motor bursts in alternation with bilaterally co-active extensor bursts. Such fictive rhythmic limb-kicks occur either independently of axial burst timing at lower frequency (A2, see randomly distributed phase values of axial VR12 versus limb L-Ext bursts in polar plot at right), or in a single co-ordinated pattern (A3; see close phase correlation between bursts of the same ventral roots as in A2 plotted at right. In the two polar plots, the concentration of phase values around the mean is denoted by the length of vector $r$ which can vary from 0 to 1 ). Note that even during the simultaneous expression of separate axial and limb rhythms (as in A2), bursts in one rhythm may be weakly modulated in time with the other, indicating a persistent functional overlap of the underlying neural circuitry. B1. At stage 64, the tail has completely regressed and locomotion is purely limb-based. B2. In correspondence, the isolated brainstem/spinal cord (see lower B1) spontaneously produces fictive limb kicks with synchronous bursts in left and right extensor motoneurons alternating with left and right flexor bursts.

Figure 4. Summary of changes in co-ordination within and between limb and tail motor patterns during Xenopus metamorphosis. A. Intra-limb co-ordination of extensor and flexor muscles switches from synchrony during pro-metamorphosis (A1) to alternation at metamorphic climax (A2). B. In contrast, inter-limb co-ordination (here represented by left and right extensor activity) switches from alternation (B1) to synchrony (B2). C. Axial and limb motor bursts, which show a strict 1:1 co-ordination (and homolateral synchrony) during pro-metamorphosis (C1), become progressively dissociated into two distinct rhythms at metamorphic climax (C2). At this critical developmental stage, however, the two spontaneous rhythms can also be associated in a single co-ordinated pattern (C3). The neuroamines 5-HT and NA are able to modulate the coupling between 
the two locomotor networks (see arrows); bath-applied 5HT produces rhythm coupling, while NA dissociates network activity into two independent rhythms.

\section{Figure 5.}

Comparison of locomotory transformations in different metamorphosing animals. A. In the anuran amphibian Xenopus laevis, larval axial-based swimming is replaced by limbbased swimming in the adult. The metamorphic transition is progressive with the animal remaining active throughout. $\mathrm{B}$. In the urodele amphibian, Pleurodeles Waltii, the limbs are also progressively added during metamorphosis, but the tail is conserved in adulthood and the animal has the capacity for either undulatory tail-based swimming or quadrupedal walking. C. In contrast, the holometabolous hawkmoth, Manduca, undergoes a complete change in body format via an immobile pupal stage involving a transformation from crawling larva to walking/flying adult. D. In the hemimetabolous locust, wings are added during the progressive metamorphic transition from the flightless nymph to the bi-modal walking/flying adult. 


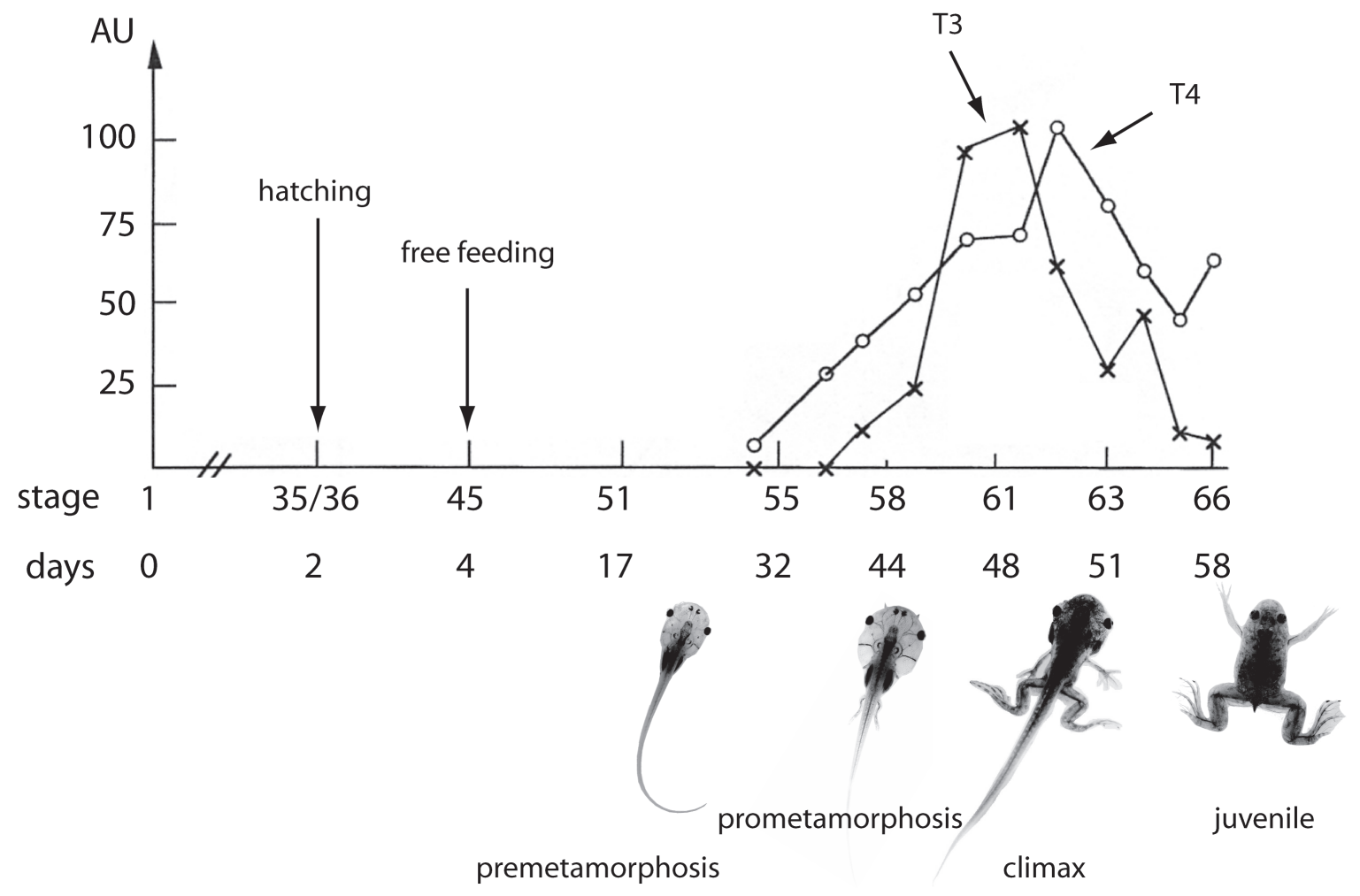

FIGURE 1 
A1 Stage 50
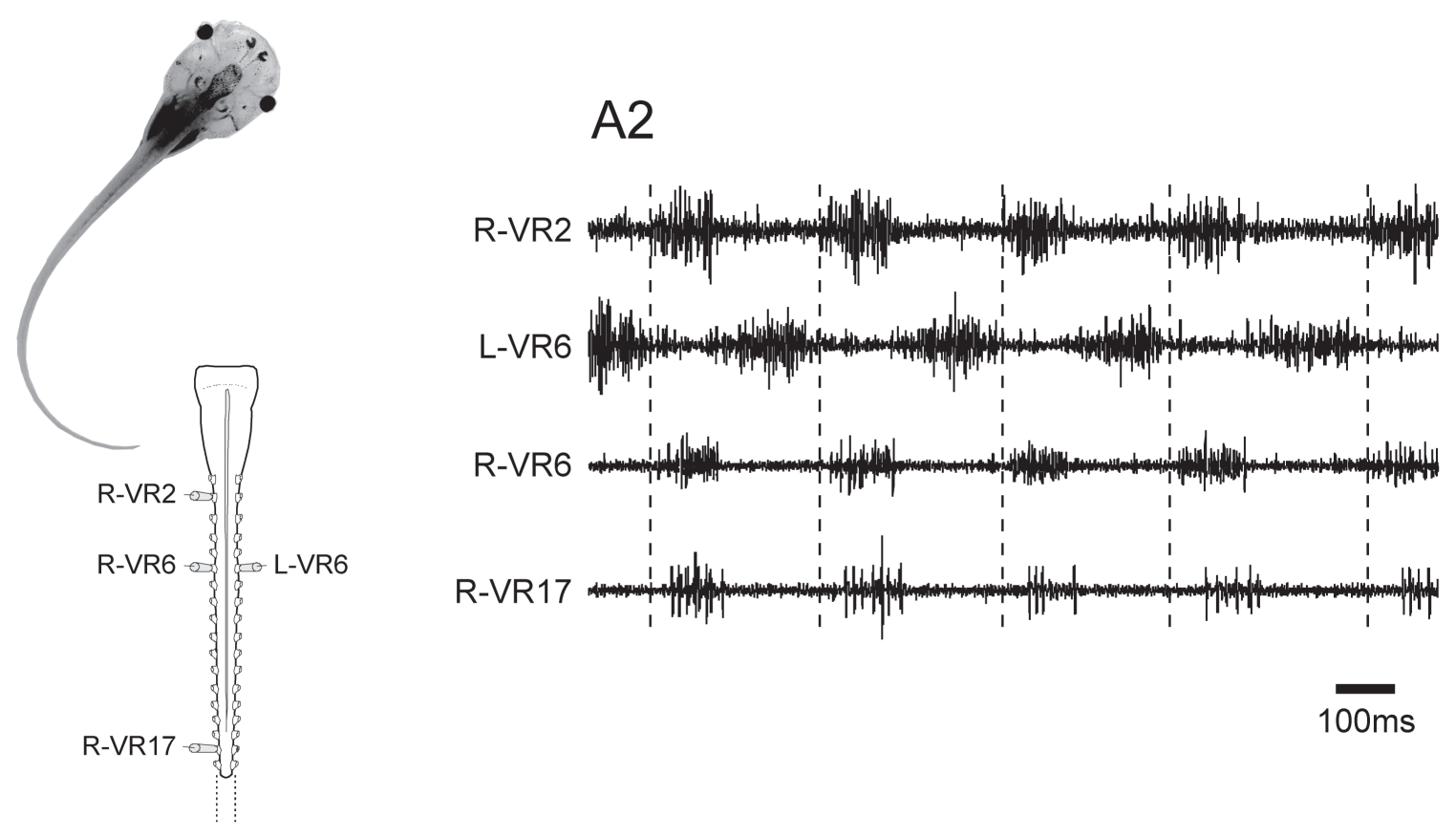

B1 Stage 58

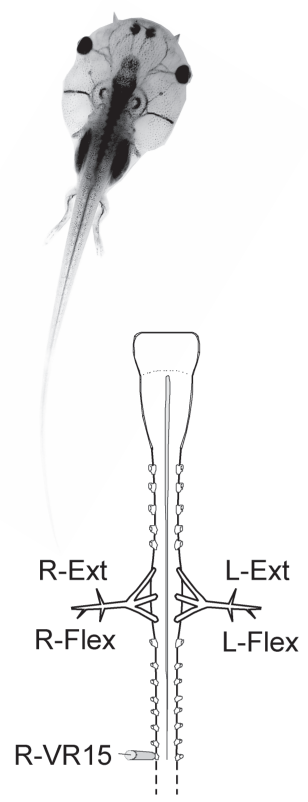

B2

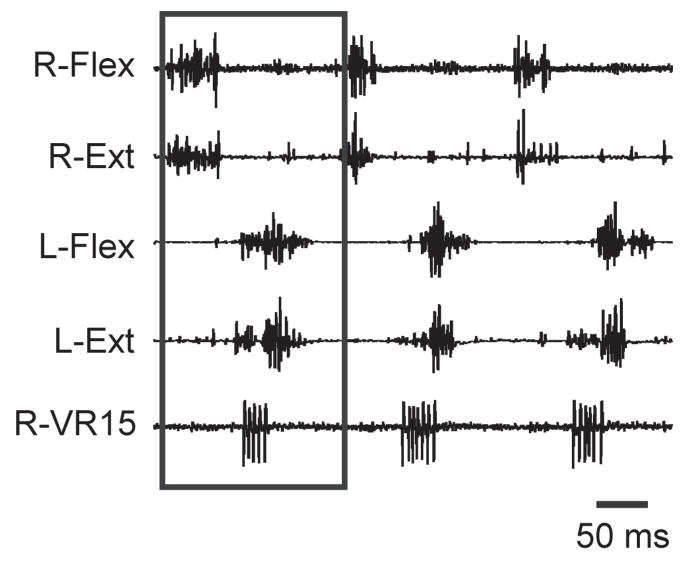

FIGURE 2 
A1 Stage 61

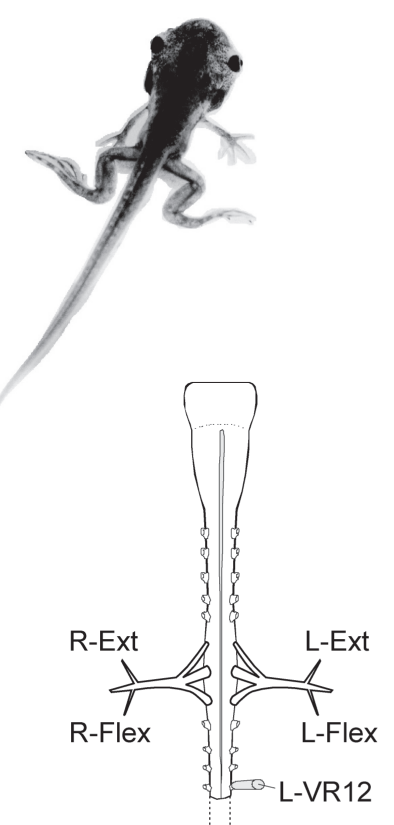

B1 Stage 64

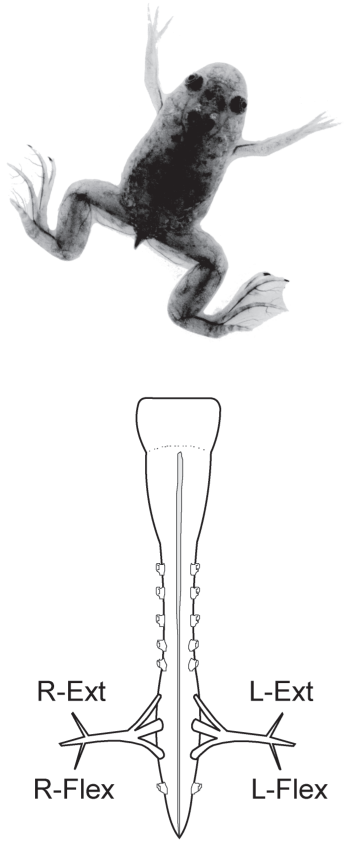

A2
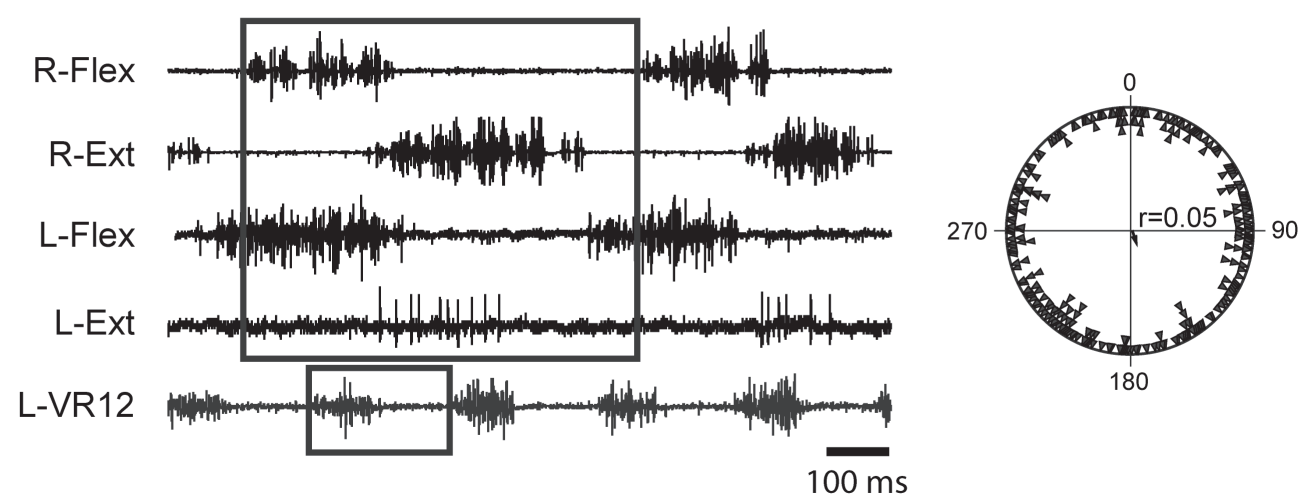

A3
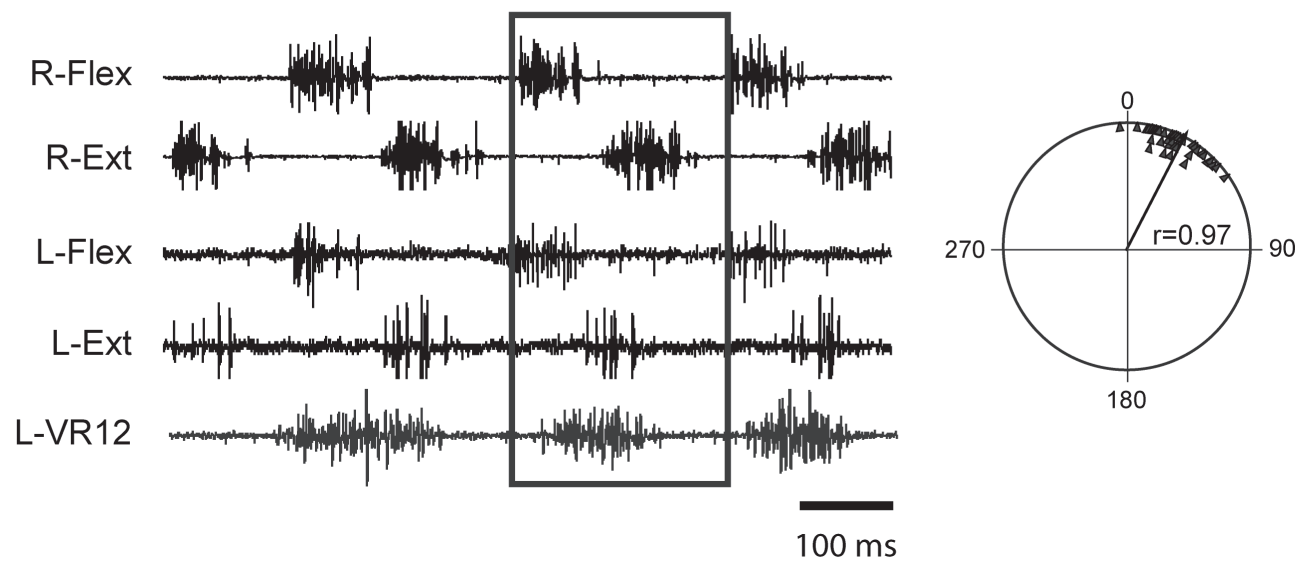

\section{B2}

R-Flex

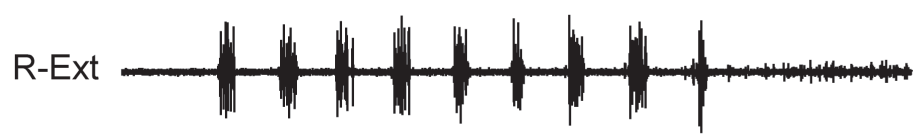

L-Flex

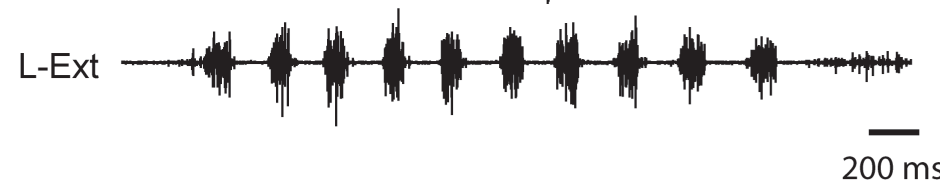




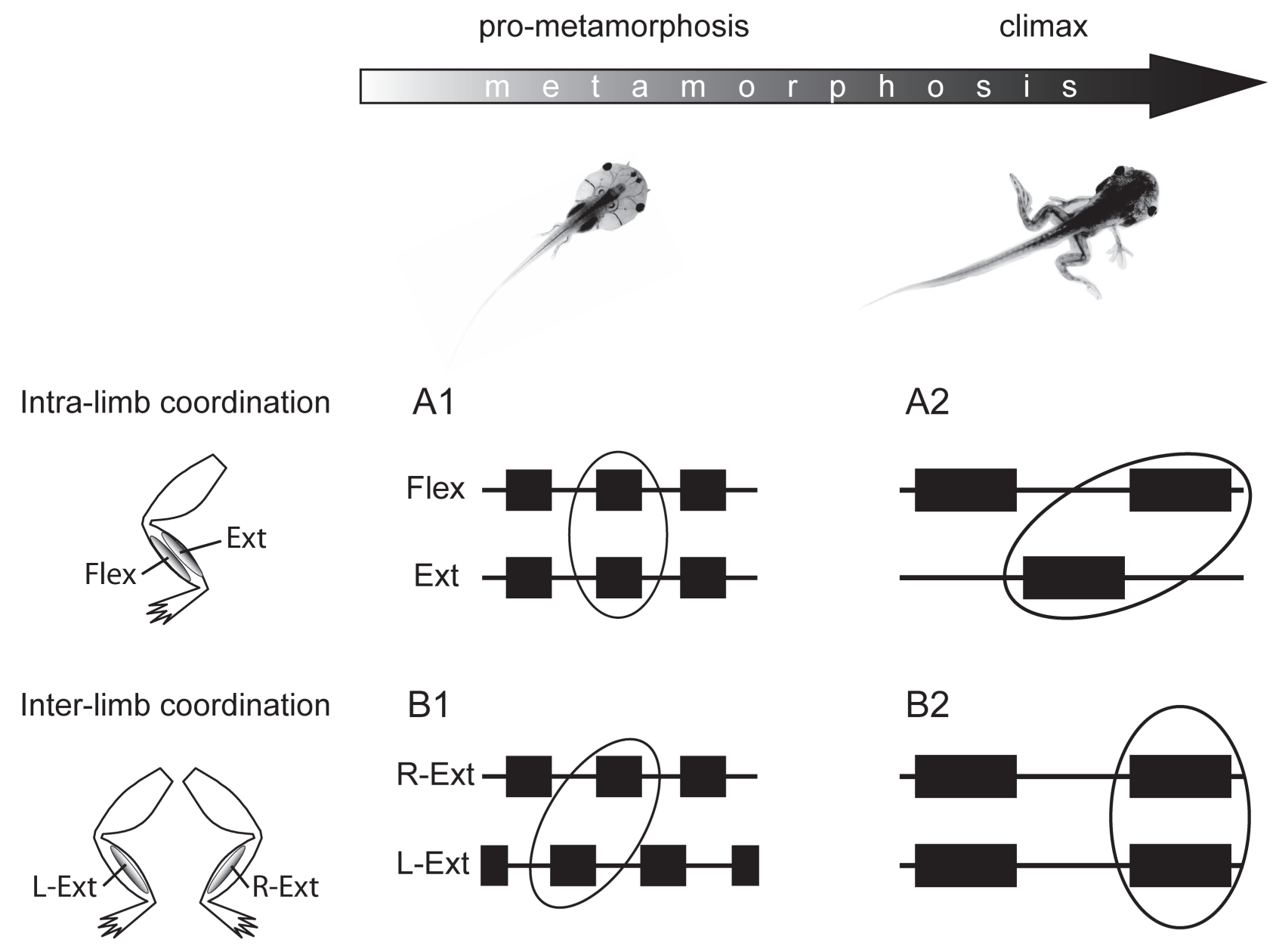

Axial-Limb coordination

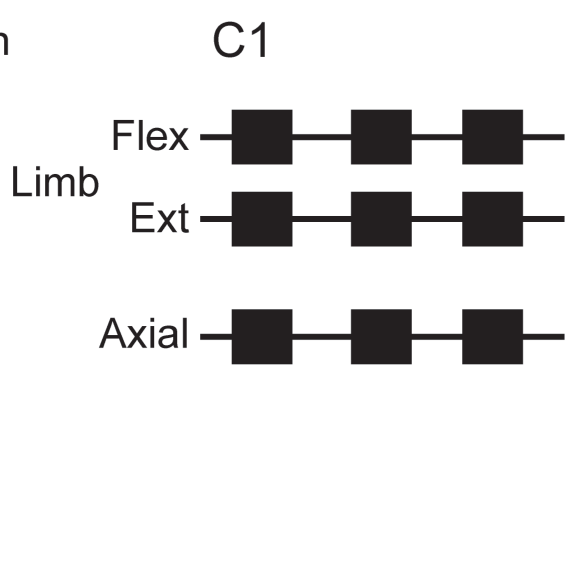

C2
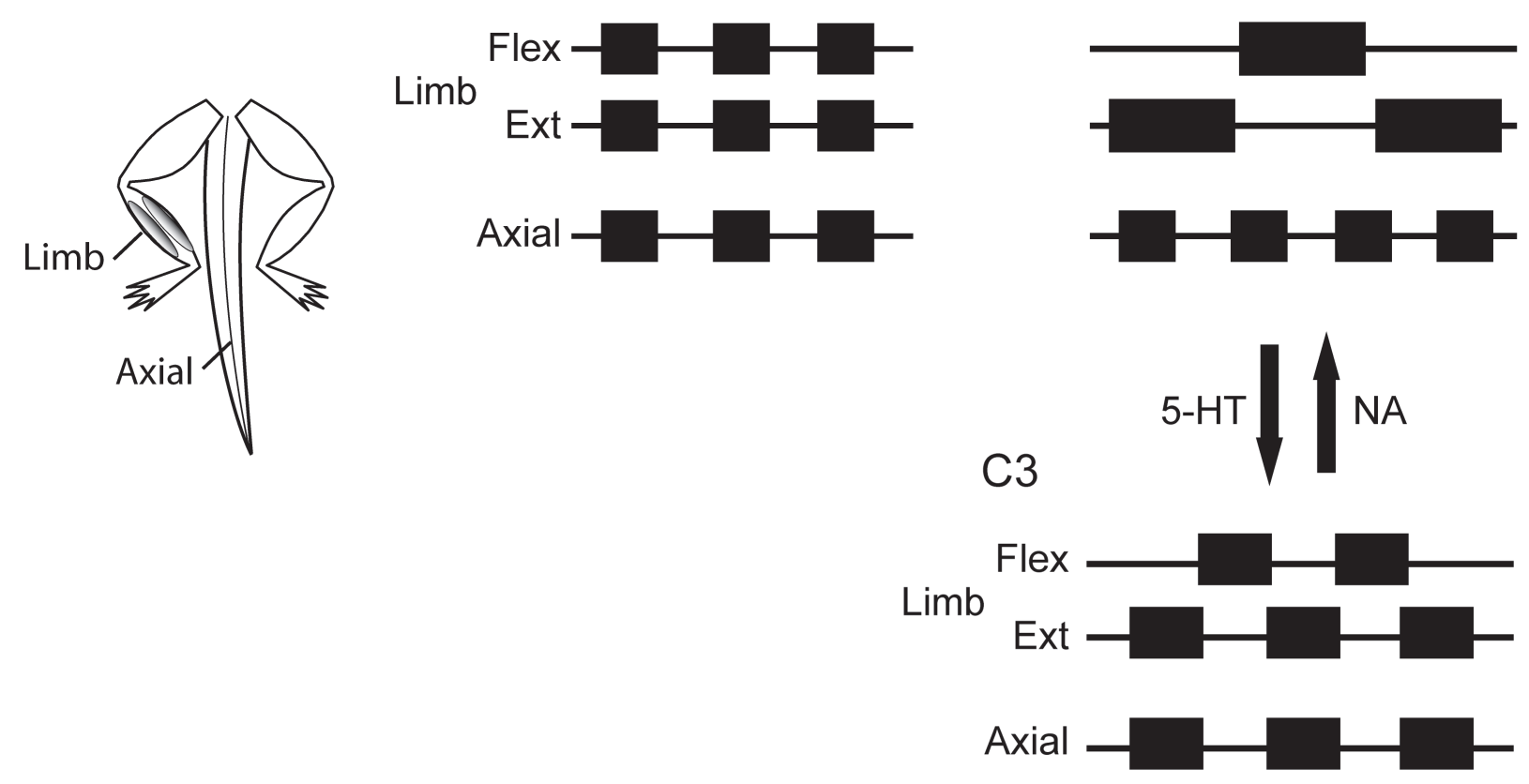

FIGURE 4 
A Xenopus

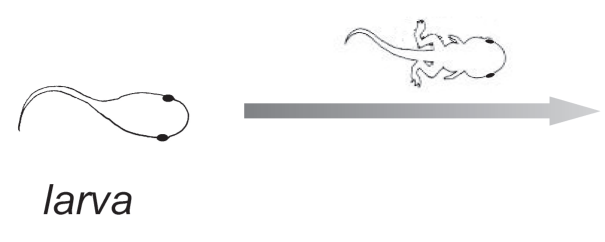

axial-based swimming

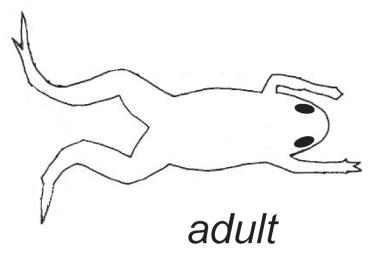

limb-based swimming
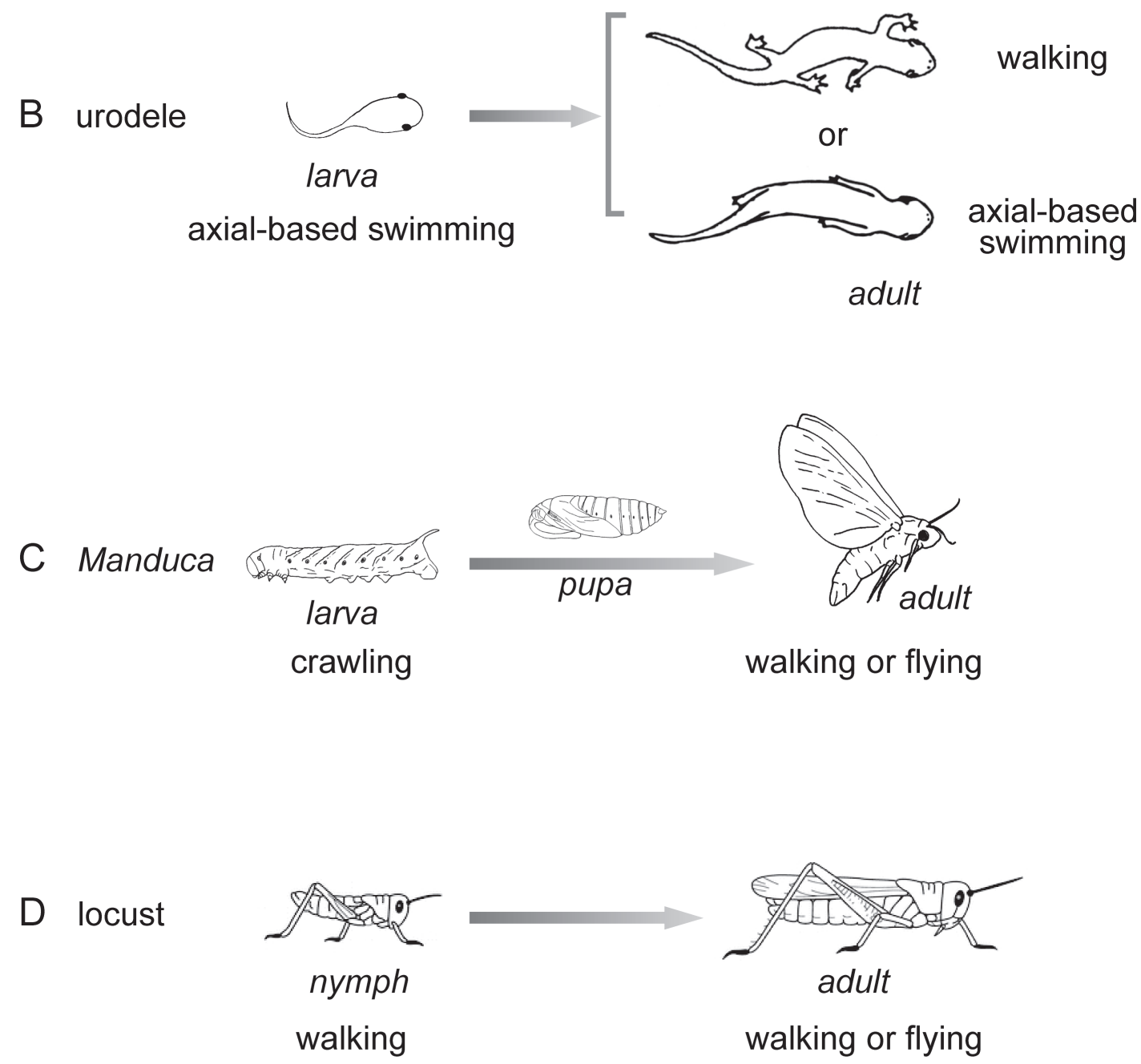

FIGURE 5 